UDK 517.5

\author{
V. A. Kofanov*, I. V. Popovich** \\ * Oles Honchar Dnipro National University, \\ Dnipro 49050.E-mail: vladimir.kofanov@gmail.com \\ ** Oles Honchar Dnipro National University.
}

\title{
Sharp Nagy type inequalities for the classes of functions with given quotient of the uniform norms of positive and negative parts of a function
}

Для довільних $p \in(0, \infty], \omega>0, d \geq 2 \omega$, отримана точна нерівність типу Надя

$$
\left\|x_{ \pm}\right\|_{\infty} \leq \frac{\left\|(\varphi+c)_{ \pm}\right\|_{\infty}}{\|\varphi+c\|_{L_{p}\left(I_{2 \omega}\right)}}\|x\|_{L_{p}\left(I_{d}\right)}
$$

на класах $S_{\varphi}(\omega) d$-періодичних функцій $x$, що мають нулі, із заданою синусоподібною $2 \omega$-періодичною функцією порівняння $\varphi$, де $c \in\left[-\|\varphi\|_{\infty},\|\varphi\|_{\infty}\right]$ задовольняє умову

$$
\left\|x_{+}\right\|_{\infty} \cdot\left\|x_{-}\right\|_{\infty}^{-1}=\left\|(\varphi+c)_{+}\right\|_{\infty} \cdot\left\|(\varphi+c)_{-}\right\|_{\infty}^{-1} .
$$

Як наслідок доведена точна нерівнсть

$$
\left\|x_{ \pm}\right\|_{\infty} \leq \frac{\left\|\left(\varphi_{r}+c\right)_{ \pm}\right\|_{\infty}}{\left\|\varphi_{r}+c\right\|_{L_{p}\left(I_{2 \pi}\right)}^{\alpha}}\|x\|_{L_{p}\left(I_{2 \pi}\right)}^{\alpha}\left\|x^{(r)}\right\|_{\infty}^{1-\alpha}, \quad \alpha=r(r+1 / p)^{-1}
$$

на соболєвських класах диференційовних періодичних функцій із заданим відношенням рівномірних норм додатної і від'ємної частин функції, де $\varphi_{r}-$ ідеальний сплайн Ейлера порядку $r$. Крім того, з нерівності (1) виведена нерівність типу Нікольського

$$
\left\|T_{ \pm}\right\|_{\infty} \leq\left(\frac{n}{m}\right)^{1 / p} \frac{\left\|(\sin (\cdot)+c)_{ \pm}\right\|_{\infty}}{\|\sin (\cdot)+c\|_{L_{p}\left(I_{2 \pi}\right)}}\|T\|_{L_{p}\left(I_{2 \pi}\right)}
$$

на просторах тригонометричних поліномів $T$ порядку $\leq n$ з періодом $2 \pi / m, m \leq n$, i заданим відношенням рівномірних норм додатної i від'ємної частин, i аналогічна нерівність типу Нікольського

$$
\left\|s_{ \pm}\right\|_{\infty} \leq\left(\frac{n}{m}\right)^{1 / p} \frac{\left\|\left(\varphi_{r}+c\right)_{ \pm}\right\|_{\infty}}{\left\|\varphi_{r}+c\right\|_{L_{p}\left(I_{2 \pi}\right)}}\|s\|_{L_{p}\left(I_{2 \pi}\right)}
$$

на просторах поліноміальних сплайнів $s$ порядку $r$ мінімального дефекту з вузлами в точках $k \pi / n, k \in \mathbf{Z}$, та періодом $2 \pi / m, m \leq n$, і заданим відношенням рівномірних норм додатної і від'ємної частин.

Ключові слова: Нерівність типу Надя, клас функцій із заданою функиією порівняння, соболевсъкий клас, поліном, сплайн. 
For any $p \in(0, \infty], \omega>0, d \geq 2 \omega$, we obtain the sharp inequality of Nagy type

$$
\left\|x_{ \pm}\right\|_{\infty} \leq \frac{\left\|(\varphi+c)_{ \pm}\right\|_{\infty}}{\|\varphi+c\| \|_{L_{p}\left(I_{2 \omega}\right)}}\|x\|_{L_{p}\left(I_{d}\right)}
$$

on the set $S_{\varphi}(\omega)$ of $d$-periodic functions $x$ having zeros with given the sine-shaped $2 \omega$ periodic comparison function $\varphi$, where $c \in\left[-\|\varphi\|_{\infty},\|\varphi\|_{\infty}\right]$ is such that

$$
\left\|x_{+}\right\|_{\infty} \cdot\left\|x_{-}\right\|_{\infty}^{-1}=\left\|(\varphi+c)_{+}\right\|_{\infty} \cdot\left\|(\varphi+c)_{-}\right\|_{\infty}^{-1} .
$$

In particular, we obtain such type inequalities on the Sobolev sets of periodic functions and on the spaces of trigonometric polynomials and polynomial splines with given quotient of the norms $\left\|x_{+}\right\|_{\infty} /\left\|x_{-}\right\|_{\infty}$.

Key words: Nagy type inequality, a class of functions with given comparison function, Sobolev class of functions, polynomial, spline.

MSC2010: Pri 41A17, SEC 41A44, 42A05, 41A15

1. Introduction. Let $G \subset \mathbf{R}$. We will consider the spaces $L_{p}(G), 0<p \leq \infty$, of all measurable functions $x: G \rightarrow \mathbf{R}$ such that $\|x\|_{p}=\|x\|_{L_{p}(G)}<\infty$, where

$$
\begin{aligned}
\|x\|_{p}:= & \left(\int_{G}|x(t)|^{p} d t\right)^{1 / p}, \quad \text { if } \quad 0<p<\infty, \\
& \|x\|_{\infty}:=\text { vrai } \sup _{t \in G}|x(t)| .
\end{aligned}
$$

Let $d>0$ and $I_{d}$ denote the circle which is realized as the interval $[0, d]$ with coincident endpoints. For $r \in \mathbf{N}, G=\mathbf{R}$ or $G=I_{d}$, denote by $L_{\infty}^{r}(G)$ the space of all functions $x \in L_{\infty}(G)$ for which $x^{(r-1)}$ is locally absolutely continuous and $x^{(r)} \in L_{\infty}(G)$.

A function $f \in L_{\infty}^{1}(\mathbf{R})$ is called a comparison function for $x \in L_{\infty}^{1}(\mathbf{R})$ if there exists a constant $c \in \mathbf{R}$ satisfying

$$
\max _{t \in \mathbf{R}} x(t)=\max _{t \in \mathbf{R}} f(t)+c, \quad \min _{t \in \mathbf{R}} x(t)=\min _{t \in \mathbf{R}} f(t)+c .
$$

and from $x(\xi)=f(\eta)+c, \xi, \eta \in \mathbf{R}$, the inequality $\left|x^{\prime}(\xi)\right| \leq\left|f^{\prime}(\eta)\right|$ follows ( if corresponding derivatives exist).

Let $\omega>0$. By definition, $S$-function is a $2 \omega$-periodic function $\varphi \in L_{\infty}^{1}\left(I_{2 \omega}\right)$ that has the following properties: vanishes at 0 , is odd about 0 , is even about $\omega / 2$, is positive and concave on $(0, \omega)$, and strictly increasing on $[0, \omega / 2]$.

For $2 \omega$-periodic $S$-function $\varphi$ denote by $S_{\varphi}(\omega)$ the class of functions $x \in L_{\infty}^{1}(\mathbf{R})$ for which $\varphi$ is the comparison function. Note that the classes $S_{\varphi}(\omega)$ were considered in [1], [2]. Examples of such classes $S_{\varphi}(\omega)$ are the Sobolev classes $\left\{x \in L_{\infty}^{r}\left(I_{d}\right):\left\|x^{(r)}\right\|_{\infty} \leq 1\right\}$, the bounded subsets of the space $T_{n}$ of all trigonometric polynomials of order at most $n$, and the same subsets of the space $S_{n, r}$ of polynomial splines of order $r$ having defect 1 with knots at the points $k \pi / n, k \in \mathbf{Z}$. 
It is shown in [3] that for $p \in[1, \infty]$ and $x \in L_{\infty}^{r}\left(I_{2 \pi}\right)$ there holds the following sharp inequality of Nagy type

$$
E_{0}(x)_{\infty} \leq \frac{\left\|\varphi_{r}\right\|_{\infty}}{\left\|\varphi_{r}\right\|_{L_{p}\left(I_{2 \pi}\right)}^{\alpha}}\|x\|_{L_{p}\left(I_{2 \pi}\right)}^{\alpha}\left\|x^{(r)}\right\|_{\infty}^{1-\alpha},
$$

where $\alpha=\frac{r}{r+1 / p}, \varphi_{r}$ is the perfect Euler spline of order $r$ and $E_{0}(x)_{\infty}$ is the best uniform approximation of the function $x$ by constants.

In this paper we generalize the inequality $(1.1)$ on the classes $S_{\varphi}(\omega)$ of a function with given quotient positive and negative parts of a function (Theorem 1). In particular, we obtain such type inequalities for a function $x \in L_{\infty}^{r}\left(I_{2 \pi}\right)$ (Theorem 2) and for functions in spaces $T_{n}$ and $S_{n, r}$ (Theorem 3 and Theorem 4) with given quotient $\left\|x_{+}\right\|_{\infty} /\left\|x_{-}\right\|_{\infty}$.

2. The inequalities of various metrics on the classes of the functions with given comparison function. For a function $f \in L_{1}\left(I_{d}\right)$ denote by $m(f, y), y>0$, the distribution function defined below

$$
m(f, y):=\mu\left\{t \in I_{d}:|f(t)|>y\right\}
$$

and let $r(f, t)$ be decreasing rearrangement (see, for example, $[4, \S 1.3]$ ) of the restriction of the function $|f|$ on $[0, d]$. Set $r(f, t)=0$ for $t>d$.

Theorem 1. Let $p \in(0, \infty]$ and $\varphi$ is $2 \omega$-periodic $S$-function. For any d-periodic function $x \in S_{\varphi}(\omega)$ having zeros the inequality

$$
\left\|x_{ \pm}\right\|_{\infty} \leq \frac{\left\|(\varphi+c)_{ \pm}\right\|_{\infty}}{\|\varphi+c\|_{L_{p}\left(I_{2} \omega\right)}}\|x\|_{L_{p}\left(I_{d}\right)}
$$

holds true, where the point $c=c(x) \in\left[-\|\varphi\|_{\infty},\|\varphi\|_{\infty}\right]$ satisfies the condition

$$
\frac{\left\|x_{+}\right\|_{\infty}}{\left\|x_{-}\right\|_{\infty}}=\frac{\left\|(\varphi+c)_{+}\right\|_{\infty}}{\left\|(\varphi+c)_{-}\right\|_{\infty}} .
$$

The inequality (2.2) is sharp on the classes of functions $x \in S_{\varphi}(\omega)$ having zeros with given quotient $\left\|x_{+}\right\|_{\infty} /\left\|x_{-}\right\|_{\infty}$ and becomes equality for the function $x(t)=\varphi(t)+c$.

Proof. Fix any $d$-periodic function $x \in S_{\varphi}(\omega)$ having zeros. Since $\varphi$ is comparison function for $x$, then there exists a constant $c \in \mathbf{R}$ satisfying

$$
\left\|x_{+}\right\|_{\infty}=\left\|(\varphi+c)_{+}\right\|_{\infty}, \quad\left\|x_{-}\right\|_{\infty}=\left\|(\varphi+c)_{-}\right\|_{\infty} .
$$

By definition, the function $\varphi$ is strictly increasing on $\left[-\frac{\omega}{2}, \frac{\omega}{2}\right]$. For $\tau \in \mathbf{R}$ set $x_{\tau}(t):=$ $x(\tau+t), t \in \mathbf{R}$. Choose $\tau_{1}, \tau_{2} \in \mathbf{R}$ such that

$$
x_{\tau_{1}}\left(\frac{\omega}{2}\right)=\left\|x_{+}\right\|_{\infty}, \quad x_{\tau_{2}}\left(-\frac{\omega}{2}\right)=\left\|x_{-}\right\|_{\infty} .
$$


Since $\varphi$ is comparison function for $x$, then

$$
\left(x_{\tau_{1}}(t)\right)_{+} \geq(\varphi(t)+c)_{+}, \quad\left|t-\frac{\omega}{2}\right| \leq \omega
$$

and

$$
\left(x_{\tau_{2}}(t)\right)_{-} \geq(\varphi(t)+c)_{-}, \quad\left|t+\frac{\omega}{2}\right| \leq \omega
$$

where $u_{ \pm}:=\max \{ \pm u, 0\}$. Observe that from (2.4) and (2.5) follows the inequalities $d \geq 2 \omega$ and

$$
m\left(x_{ \pm}, y\right) \geq m\left((\varphi(\cdot)+c)_{ \pm}, y\right), \quad y \geq 0,
$$

where the function $m(f, y)$ is defined by (2.1). It follows immediately that

$$
r(x, t) \geq r(\varphi(\cdot)+c, t), \quad t \geq 0 .
$$

Combining the inequalities (2.6) and $d \geq 2 \omega$ we have

$$
\|x\|_{L_{p}\left(I_{d}\right)}^{p}=\int_{0}^{2 d} r^{p}(x, t) d t \geq \int_{0}^{2 \omega} r^{p}(\varphi(\cdot)+c, t) d t=\int_{I_{2 \omega}}|\varphi(t)+c|^{p} d t .
$$

Therefore,

$$
\|x\|_{L_{p}\left(I_{d}\right)} \geq\|\varphi+c\|_{L_{p}\left(I_{2 \omega}\right.} .
$$

It yields (2.2) in view of (2.3). Theorem 1 is proved.

Denote by $E_{0}(f)_{L_{p}(G)}$ the best approximation of the function $f$ by constants in the space $L_{p}(G)$ and let

$$
E_{0}^{ \pm}(x)_{L_{p}(G)}:=\inf _{c \in \mathbf{R}}\left\{\|x-c\|_{L_{p}(G)}: \forall t \pm(x(t)-c)_{ \pm} \geq 0\right\}
$$

be the best one-sided approximations by constants of the function $f$ in that space.

Corollary 1. Under the assumptions of Theorem 1 for any $x \in S_{\varphi}(\omega)$

$$
E_{0}(x)_{\infty} \leq \frac{\|\varphi\|_{\infty}}{E_{0}(\varphi)_{L_{p}\left(I_{2 \omega}\right)}}\|x\|_{L_{p}\left(I_{d}\right)}
$$

and

$$
E_{0}^{ \pm}(x)_{\infty} \leq \frac{2\|\varphi\|_{\infty}}{\|\varphi+\bar{c}\|_{L_{p}\left(I_{2 \omega}\right)}} E_{0}^{ \pm}(x)_{L_{p}\left(I_{d}\right)}, \quad \bar{c}:=\|\varphi\|_{\infty} .
$$

Besides, for a function $x \in S_{\varphi}(\omega)$ having zeros the inequality

$$
\|x\|_{\infty} \leq \sup _{c:|c| \leq\|\varphi\|_{\infty}} \frac{\|\varphi+c\|_{\infty}}{\|\varphi+c\|_{L_{p}\left(I_{2 \omega}\right)}}\|x\|_{L_{p}\left(I_{d}\right)}
$$

holds true. 
3. Nagy type inequalities for differentiable periodic functions. We denote be $\varphi_{r}(t), r \in \mathbf{N}$, the shift of the $r^{t h} 2 \pi$-periodic integral with zero mean value on a period of the function $\varphi_{0}(t)=\operatorname{sgn} \sin t$ satisfying $\varphi_{r}(0)=0$ and $\varphi_{r}^{\prime}(0)>0$. Let $K_{r}:=\left\|\varphi_{r}\right\|_{\infty}$ be the Favard constant. For $\lambda>0$ set $\varphi_{\lambda, r}(t):=\lambda^{-r} \varphi_{r}(\lambda t)$. Obviously the spline $\varphi_{\lambda, r}(t)$ is $2 \pi / \lambda$-periodic $S$-function.

Theorem 2. Let $r \in \mathbf{N}, p \in(0, \infty]$. Then for any function $x \in L_{\infty}^{r}\left(I_{2 \pi}\right)$ having zeros we have

$$
\left\|x_{ \pm}\right\|_{\infty} \leq \frac{\left\|\left(\varphi_{r}+c\right)_{ \pm}\right\|_{\infty}}{\left\|\varphi_{r}+c\right\|_{L_{p}\left(I_{2 \pi}\right)}^{\alpha}}\|x\|_{L_{p}\left(I_{2 \pi}\right)}^{\alpha}\left\|x^{(r)}\right\|_{\infty}^{1-\alpha}
$$

where $\alpha=\frac{r}{r+1 / p}$ and $c \in\left[-K_{r}, K_{r}\right]$ is such that

$$
\frac{\left\|x_{+}\right\|_{\infty}}{\left\|x_{-}\right\|_{\infty}}=\frac{\left\|\left(\varphi_{r}+c\right)_{+}\right\|_{\infty}}{\left\|\left(\varphi_{r}+c\right)_{-}\right\|_{\infty}}
$$

The inequality (3.1) is sharp on the class of functions $x \in L_{\infty}^{r}\left(I_{2 \pi}\right)$ having zeros with given quotient $\left\|x_{+}\right\|_{\infty} /\left\|x_{-}\right\|_{\infty}$ and becomes equality for the function $x(t)=\varphi_{r}(t)+c$.

Proof. Fix a function $x \in L_{\infty}^{r}(\mathbf{R})$ having zeros. In view of homogeneity of the inequality (3.1) we can assume that

$$
\left\|x^{(r)}\right\|_{\infty}=1
$$

Choose $\lambda$ satisfying

$$
E_{0}(x)_{\infty}=\left\|\varphi_{\lambda, r}\right\|_{\infty}
$$

Then by the Kolmogorov comparison theorem [5] the spline $\varphi:=\varphi_{\lambda, r}$ is the comparison function for the function $x$. Consequently $x \in S_{\varphi}\left(\frac{\pi}{\lambda}\right)$, and by Theorem 1 we have

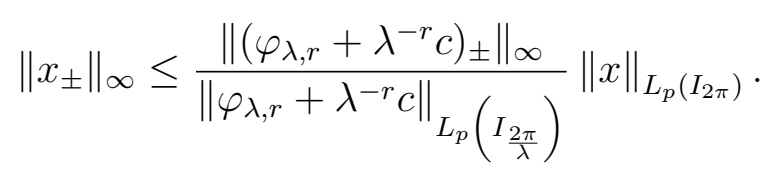

Besides, it follows from (3.3) in view of condition of Theorem 2 for constant $c$ that

$$
\left\|x_{ \pm}\right\|_{\infty}=\left\|\left(\varphi_{\lambda, r}+\lambda^{-r} c\right)_{ \pm}\right\|_{\infty}=\lambda^{-r}\left\|\left(\varphi_{r}+c\right)_{ \pm}\right\|_{\infty} .
$$

Combining (3.4) and (3.5) we get

$$
\|x\|_{L_{p}\left(I_{2 \pi}\right)} \geq\left\|\varphi_{\lambda, r}+\lambda^{-r} c\right\|_{L_{p}\left(I_{\frac{2 \pi}{\lambda}}\right)}=\lambda^{-(r+1 / p)}\left\|\varphi_{r}+c\right\|_{L_{p}\left(I_{2 \pi}\right)} .
$$

Applying (3.5), (3.6) and taking into account the definition of $\alpha$ we obtain

$$
\frac{\left\|x_{ \pm}\right\|_{\infty}}{\|x\|_{L_{p}\left(I_{2 \pi}\right)}^{\alpha}} \leq \frac{\lambda^{-r}\left\|\left(\varphi_{r}+c\right)_{ \pm}\right\|_{\infty}}{\lambda^{-(r+1 / p) \alpha}\left\|\varphi_{r}+c\right\|_{L_{p}\left(I_{2 \pi}\right)}^{\alpha}}=\frac{\left\|\left(\varphi_{r}+c\right)_{ \pm}\right\|_{\infty}}{\left\|\varphi_{r}+c\right\|_{L_{p}\left(I_{2 \pi}\right)}^{\alpha}} .
$$

It follows (3.1) in view of (3.2). Theorem 2 is proved. 
Corollary 2. Under the assumptions of Theorem 1 for any $x \in L_{\infty}^{r}\left(I_{2 \pi}\right)$

$$
E_{0}(x)_{\infty} \leq \frac{K_{r}}{E_{0}\left(\varphi_{r}\right)_{L_{p}\left(I_{2 \pi}\right)}^{\alpha}}\|x\|_{L_{p}\left(I_{2 \pi}\right)}^{\alpha}\left\|x^{(r)}\right\|_{\infty}^{1-\alpha}
$$

and

$$
E_{0}^{ \pm}(x)_{\infty} \leq \frac{2 K_{r}}{\left\|\varphi_{r}+K_{r}\right\|_{L_{p}\left(I_{2 \pi}\right)}^{\alpha}} E_{0}^{ \pm}(x)_{L_{p}\left(I_{2 \pi}\right)}^{\alpha}\left\|x^{(r)}\right\|_{\infty}^{1-\alpha},
$$

Besides, for a function $x \in L_{\infty}^{r}\left(I_{2 \pi}\right)$ having zeros the inequality

$$
\|x\|_{\infty} \leq \sup _{c:|c| \leq K_{r}} \frac{\left\|\varphi_{r}+c\right\|_{\infty}}{\left\|\varphi_{r}+c\right\|_{L_{p}\left(I_{2 \pi}\right)}^{\alpha}}\|x\|_{L_{p}\left(I_{2 \pi}\right)}^{\alpha}\left\|x^{(r)}\right\|_{\infty}^{1-\alpha} .
$$

holds true.

Remark 2. The first inequality is proved in [3] and the rest ones are proved in [6].

4. Nikolskii type inequalities for trigonometric polynomials. Let us recall that $T_{n}$ is the space of all trigonometric polynomials of degree at most $n$.

Theorem 3. Let $p \in(0, \infty], n, m \in \mathbf{N}, m \leq n$. For any trigonometric polynomial $T \in T_{n}$ with minimal period $2 \pi / m$ having zeros the inequality

$$
\left\|T_{ \pm}\right\|_{\infty} \leq\left(\frac{n}{m}\right)^{1 / p} \frac{\left\|(\sin (\cdot)+c)_{ \pm}\right\|_{\infty}}{\|\sin (\cdot)+c\|_{L_{p}\left(I_{2 \pi}\right)}}\|T\|_{L_{p}\left(I_{2 \pi}\right)}
$$

holds true, where $c \in[-1,1]$ is the constant satisfying

$$
\frac{\left\|T_{+}\right\|_{\infty}}{\left\|T_{-}\right\|_{\infty}}=\frac{\left\|(\sin (\cdot)+c)_{+}\right\|_{\infty}}{\left\|(\sin (\cdot)+c)_{-}\right\|_{\infty}} .
$$

The inequality (4.1) is sharp for $m=1$ in the sense

$$
\sup _{n \in \mathbf{N}} \sup _{T \in T_{n}(c)} \frac{\left\|T_{ \pm}\right\|_{\infty}}{n^{1 / p}\|T\|_{L_{p}\left(I_{2 \pi}\right)}}=\frac{\left\|(\sin (\cdot)+c)_{ \pm}\right\|_{\infty}}{\|\sin (\cdot)+c\|_{L_{p}\left(I_{2 \pi}\right)}},
$$

where $T_{n}(c)$ is the set of all trigonometric polynomials $T \in T_{n}$ having zeros with given quotient $\left\|T_{+}\right\|_{\infty} /\left\|T_{-}\right\|_{\infty}$ satisfying (4.2).

Proof. Fix a polynomial $T \in T_{n}$ with minimal period $\frac{2 \pi}{m}$ having zeros. In view of homogeneity of the inequality (4.1) we can assume that

$$
E_{0}(T)_{\infty}=1
$$

Then the polynomial $\varphi(t):=\sin n t$ is comparison function for the polynomial $T(t)$ (see, for example, the proof of Theorem 8.1.1 [7]). It is clear that $\varphi$ is $\frac{2 \pi}{n}$-periodic $S$-function. Hence $T \in S_{\varphi}\left(\frac{\pi}{n}\right)$. Then by Theorem 1

$$
\left\|T_{ \pm}\right\|_{\infty} \leq \frac{\left\|(\sin n(\cdot)+c)_{ \pm}\right\|_{\infty}}{\|\sin n(\cdot)+c\|_{L_{p}\left(I_{\frac{2 \pi}{n}}\right)}}\|T\|_{L_{p}\left(I_{\frac{2 \pi}{m}}\right)} .
$$


Inequality (4.1) follows in view of the evident equalities

$$
\begin{gathered}
\|T\|_{L_{p}\left(I_{\frac{2 \pi}{m}}\right)}=m^{-1 / p}\|T\|_{L_{p}\left(I_{2 \pi}\right)}, \\
\|\sin n(\cdot)+c\|_{L_{p}\left(I_{\frac{2 \pi}{n}}\right)}=n^{-1 / p}\|\sin (\cdot)+c\|_{L_{p}\left(I_{2 \pi}\right)}
\end{gathered}
$$

and

$$
\left\|(\sin n(\cdot)+c)_{ \pm}\right\|_{\infty}=\left\|(\sin (\cdot)+c)_{ \pm}\right\|_{\infty}
$$

Theorem 3 is proved.

Corollary 3. Under the assumptions of Theorem 3 for a polynomial $T \in T_{n}$ with minimal period $2 \pi / m$ we have

$$
E_{0}(T)_{\infty} \leq\left(\frac{n}{m}\right)^{1 / p} \frac{\|T\|_{L_{p}\left(I_{2 \pi}\right)}}{E_{0}(\sin (\cdot))_{L_{p}\left(I_{2 \pi}\right)}}
$$

and

$$
E_{0}^{ \pm}(T)_{\infty} \leq 2\left(\frac{n}{m}\right)^{1 / p} \frac{E_{0}^{ \pm}(T)_{L_{p}\left(I_{2 \pi}\right)}}{\|\sin (\cdot)+1\|_{L_{p}\left(I_{2 \pi}\right)}} .
$$

Besides, for a polynomial $T \in T_{n}$ with minimal period $2 \pi / m$ having zeros

$$
\|T\|_{\infty} \leq\left(\frac{n}{m}\right)^{1 / p} \sup _{c:|c| \leq 1} \frac{\|\sin (\cdot)+c\|_{\infty}}{\|\sin (\cdot)+c\|_{L_{p}\left(I_{2 \pi}\right)}}\|T\|_{L_{p}\left(I_{2 \pi}\right)} .
$$

Remark 3. For $m=1$ the first inequality is proved in [3] and the rest ones are proved in [6].

5. Nikolskii type inequalities for periodic polynomial splines. Let $r, n \in \mathbf{N}$. Recall that $S_{n, r}$ stands for the space of polynomial splines of order $r$ having defect 1 with knots at the points $k \pi / n, k \in \mathbf{Z}$. It is clear that $S_{n, r} \subset L_{\infty}^{r}(\mathbf{R})$.

Theorem 4. Let $p \in(0, \infty] ; n, m \in \mathbf{N}, m \leq n$. For a spline $s \in S_{n, r}$ with minimal period $2 \pi / m$ having zeros the inequality

$$
\left\|s_{ \pm}\right\|_{\infty} \leq\left(\frac{n}{m}\right)^{1 / p} \frac{\left\|\left(\varphi_{r}+c\right)_{ \pm}\right\|_{\infty}}{\left\|\varphi_{r}+c\right\|_{L_{p}\left(I_{2 \pi}\right)}}\|s\|_{L_{p}\left(I_{2 \pi}\right)}
$$

holds true, where $c \in\left[-K_{r}, K_{r}\right]$ is the constant satisfying

$$
\frac{\left\|s_{+}\right\|_{\infty}}{\left\|s_{-}\right\|_{\infty}}=\frac{\left\|\left(\varphi_{r}+c\right)_{+}\right\|_{\infty}}{\left\|\left(\varphi_{r}+c\right)_{-}\right\|_{\infty}} .
$$

The inequality (5.1) is sharp for $m=1$ in the sense

$$
\sup _{n \in \mathbf{N}} \sup _{s \in S_{n, r}(c)} \frac{\left\|s_{ \pm}\right\|_{\infty}}{n^{1 / p}\|s\|_{L_{p}\left(I_{2 \pi}\right)}}=\frac{\left\|\left(\varphi_{r}+c\right)_{ \pm}\right\|_{\infty}}{\left\|\varphi_{r}+c\right\|_{L_{p}\left(I_{2 \pi}\right)}},
$$

where $S_{n, r}(c)$ is the set of all splines $s \in S_{n, r}$ having zeros with given quotient $\left\|s_{+}\right\|_{\infty} /\left\|s_{-}\right\|_{\infty}$ satisfying (5.2). 
Proof. Fix a spline $s \in S_{n, r}$ with minimal period $2 \pi / m$ having zeros. In view of homogeneity of the inequality (5.1) we can assume that

$$
E_{0}(s)_{\infty}=\left\|\varphi_{n, r}\right\|_{\infty}
$$

Then by the Tikhomirov inequality [8]

$$
\left\|s^{(r)}\right\|_{\infty} \leq \frac{E_{0}(s)_{\infty}}{\left\|\varphi_{n, r}\right\|_{\infty}}=1
$$

Hence all conditions of Kolmogorov comparison theorem [5] are fulfilled. By this Theorem the function $\varphi(t):=\varphi_{n, r}(t)$ is comparison function for the spline $s$. It is clear that $\varphi$ is $S$-function with period $2 \pi / n$. So $s \in S_{\varphi}\left(\frac{\pi}{n}\right)$. Then by Theorem 1

$$
\left\|s_{ \pm}\right\|_{\infty} \leq \frac{\left\|\left(\varphi_{n, r}+n^{-r} c\right)_{ \pm}\right\|_{\infty}}{\left\|\varphi_{n, r}+n^{-r} c\right\|_{L_{p}\left(I_{\frac{2 \pi}{n}}\right)}}\|s\|_{L_{p}\left(I_{\frac{2 \pi}{m}}\right)} .
$$

(5.1) follows in view of the evident equalities

$$
\begin{gathered}
\|s\|_{L_{p}\left(I_{\frac{2 \pi}{m}}\right)}=m^{-1 / p}\|s\|_{L_{p}\left(I_{2 \pi}\right)} \\
\left\|\varphi_{n, r}+n^{-r} c\right\|_{L_{p}\left(I_{\frac{2 \pi}{n}}\right)}=n^{-(r+1 / p)}\left\|\varphi_{r}+c\right\|_{L_{p}\left(I_{2 \pi}\right)}
\end{gathered}
$$

and

$$
\left\|\left(\varphi_{n, r}+n^{-r} c\right)_{ \pm}\right\|_{\infty}=n^{-r}\left\|\left(\varphi_{r}+c\right)_{ \pm}\right\|_{\infty} .
$$

Theorem 4 is proved.

Corollary 4. By conditions of Theorem 4 for a spline $s \in S_{n, r}$ with minimal period $2 \pi / m$ we have

$$
E_{0}(s)_{\infty} \leq\left(\frac{n}{m}\right)^{1 / p} \frac{K_{r}}{E_{0}\left(\varphi_{r}\right)_{L_{p}\left(I_{2 \pi}\right)}}\|s\|_{L_{p}\left(I_{2 \pi}\right)}
$$

and

$$
E_{0}^{ \pm}(s)_{\infty} \leq\left(\frac{n}{m}\right)^{1 / p} \frac{2 K_{r}}{\left\|\varphi_{r}+K_{r}\right\|_{L_{p}\left(I_{2 \pi}\right)}} E_{0}^{ \pm}(s)_{L_{p}\left(I_{2 \pi}\right)} .
$$

Besides, for a spline $s \in S_{n, r}$ with minimal period $2 \pi / m$ having zeros

$$
\|s\|_{\infty} \leq\left(\frac{n}{m}\right)^{1 / p} \sup _{c:|c| \leq K_{r}} \frac{\left\|\varphi_{r}+c\right\|_{\infty}}{\left\|\varphi_{r}+c\right\|_{L_{p}\left(I_{2 \pi}\right)}}\|s\|_{L_{p}\left(I_{2 \pi}\right)} .
$$

Remark 4. For $m=1$ the first inequality is proved in [3] and the rest ones are proved in [6].

\section{References}

1. Bojanov B. An extension of the Landau-Kolmogorov inequality. Solution of a problem of Erdos [Text] / B. Bojanov, N. Naidenov// Journal d'Analyse Mathematique. - 1999. 78. - P. $263-280$. 
2. Кофанов B. A. Точные верхние грани норм функций и их производных на классах функций с заданной функцией сранения[Текст] /В. А. Кофанов // Укр. мат. журн. - 2011. - 63, 7. - C. $969-984$.

3. Babenko V. F., Kofanov V. A. and Pichugov S. A. Inequalities of Kolmogorov Type and Some Their Applications in Approximation Theory[Text] / V. F. Babenko, V. A. Kofanov and S. A. Pichugov // Rendiconti del Circolo Matematico di Palermo Serie II, Suppl.1998.- 52. - P. $223-237$.

4. Корнейчук Н. П., Бабенко В. Ф., Лигун А. А. Экстремальные свойства полиномов и сплайнов [Текст] / Н. П. Корнейчук, В. Ф. Бабенко, А. А. Лигун// К.: Наукова думка, 1992. - 304 с.

5. Колмогоров A. Н. О неравенствах между верхними гранями последовательных производных функции на бесконечном интервале [Текст] / А. Н. Колмогоров // Избр. труды. Математика, механика.- М.: Наука, 1985. - 470 с.- С. 252-263.

6. Babenko V. F., Kofanov V. A. and Pichugov S. A. Comparison of rearrangements and Kolmogorov-Nagy type inequalities for periodic functions [Text] / V. F. Babenko, V. A. Kofanov and S. A. Pichugov //Approximation Theory: A volume dedicated to Blagovest Sendov (B. Bojanov, Ed.).- Darba, Sofia, 2002. P. $24-53$.

7. Корнейчук Н. П., Бабенко В. Ф., Кофанов В. А., Пичугов С. А. Неравенства для производных и их приложения [Текст] / Н. П. Корнейчук, В. Ф. Бабенко, В. А. Кофанов, С. А. Пичугов // К.: Наукова думка, 2003. - 590 с.

8. Тихомиров В. М. Поперечники множеств в функциональных пространствах и теория наилучших приближений [Текст] /В. М. Тихомиров// Успехи мат. наук. - 1960. - 15, 3. - C. $81-120$.

Received: 14.02.2020. Accepted: 02.05.2020 